\title{
The characterization of differential operators by locality: abstract derivations
}

\author{
C. J. K. BATTY AND D. W. ROBINSON
}

Department of Mathematics, University of Edinburgh, King's Buildings, Edinburgh EH9 3JZ, Scotland; Department of Mathematics, Institute of Advanced Studies, Australian National University, GPO Box 4, Canberra, ACT 2601, Australia

(Received 1 October 1984 and revised 30 November 1984)

Abstract. Let $\delta$ be a closed $*$-derivation on a commutative $C^{*}$-algebra $\mathscr{A}$, suppose that $\mathscr{A}_{n}=\bigcap_{m=1}^{n} D\left(\delta^{m}\right)$ is dense in $\mathscr{A}$ for some $n=1,2, \ldots, \infty$, and let $H: \mathscr{A}_{n} \rightarrow \mathscr{A}$ be a linear operator satisfying the locality condition

$$
\operatorname{supp}(H f) \subseteq \operatorname{supp}(f), \quad f \in \mathscr{A}_{n} .
$$

It is shown that $H=\sum_{m=0}^{p} l_{m} \delta^{m}$ on $\mathscr{A}_{2 n}$, for some finite integer $p \leq n$ and functions $l_{m}$ on $X$. Estimates on the coefficients $l_{m}$ are obtained and applied to flows and local flows.

\section{Introduction}

In 1960 Peetre [6] obtained an abstract characterization of partial differential operators on a bounded open subset $U$ of $\mathbb{R}^{\nu}$ in terms of locality conditions. In the case $\nu=1$ he established that a linear operator $H: C_{c}^{\infty}(U) \rightarrow C_{b}(\mathbb{R})$ satisfies the locality condition

$$
\operatorname{supp}(H f) \subseteq \operatorname{supp}(f)
$$

for all $f \in C_{c}^{\infty}(U)$ if, and only if, it is a differential operator of the form

$$
(H f)(t)=\sum_{m=0}^{p} l_{m}(t) f^{(m)}(t)
$$

for some integer $p$ and continuous functions $l_{m}$. Here $C_{c}^{\infty}(U)$ denotes the infinitely often differentiable functions with compact support in $U$ and $C_{b}(\mathbb{R})$ denotes the bounded continuous functions over $\mathbb{R}$. Consequently a linear operator $H: C_{c}^{\infty}(\mathbb{R}) \rightarrow$ $C_{b}(\mathbb{R})$ satisfies the locality condition $(*)$ for all $f \in C_{c}^{\infty}(\mathbb{R})$ if, and only if, it is locally of the form $(* *)$ with continuous coefficients $l_{m}$, i.e. the order $p$ of $H$ and the $l_{m}$ are locally bounded functions over $\mathbb{R}$ which are not necessarily globally bounded.

Recently Bratteli, Elliott and one of the present authors [3] derived analogous characterizations of differential operators associated with a general flow, or local flow, and also obtained information on the growth of the corresponding coefficients $l_{m}$. For example in the above context it was shown that a linear operator $H: C_{0}^{\infty}(\mathbb{P}) \rightarrow$ $C_{b}(\mathbb{R})$ satisfies the locality condition $(*)$ for all $f \in C_{0}^{\infty}(\mathbb{R})$ if, and only if, it has the form $(* *)$ for some integer $p$ and bounded continuous functions $l_{m}$. Here $C_{0}^{\infty}(\mathbb{R})$ denotes the infinitely often differentiable functions which vanish at infinity on $\mathbb{R}$. 
More generally if $U$ is any open subset of $\mathbb{R}$ then a linear operator $H: C_{0}^{\infty}(U) \rightarrow C_{b}(\mathbb{R})$ is local if, and only if, it is a differential operator of finite order with coefficients $l_{m}(t)$ characterized by polynomial growth in the inverse of the distance of $t$ to the boundary of $U$.

In this paper we re-examine these results in the more general context of an infinitesimal flow. Specifically let $\mathscr{A}=C_{0}(X)$ denote the continuous functions which vanish at infinity on the locally compact Hausdorff space $X$ and let $\delta$ be a closed derivation of $\mathscr{A}$; then we consider linear operators $H$ defined on $\mathscr{A}_{n}=D\left(\delta^{n}\right)$, or $\mathscr{A}_{\infty}=\bigcap_{m=1}^{\infty} D\left(\delta^{m}\right)$, and show that in general circumstances locality forces $H$ to have the form

$$
H=\sum_{m=0}^{p} l_{m} \delta^{m}
$$

with continuous coefficients $l_{m}$ of controllable growth. Note that if $\delta$ is the generator of a strongly continuous one-parameter group of $*$-automorphisms $\tau$ of $\mathscr{A}$ then we recover the situation studied in [3]; the $C^{*}$-dynamical system $(\mathscr{A}, \tau, \mathbb{R})$ determines a classical dynamical system $(X, T, \mathbb{R})$ where the flow $(T, \mathbb{R})$ satisfies $f\left(T_{t} \omega\right)=$ $\left(\tau_{t} f\right)(\omega)$ for all $f \in C_{0}(X), \omega \in X$, and $t \in \mathbb{R}$.

A special case of these problems was examined in [1] (see also the summary [2]). In particular a study was made of derivations $H$ defined on $D(\delta)$, or $\mathscr{A}_{\infty}$, and it was shown that $H=l_{1} \delta$ for some function $l_{1}$. But one can establish that a derivation defined on $D(\delta)$, or $\mathscr{A}_{\infty}$, is automatically local and then this result is a special case of the results of this paper.

The contents of the paper are summarized as follows. In $\$ 2$ we prove automatic continuity of local operators $H: \mathscr{A}_{n} \rightarrow \mathscr{A}$ by combining the proof of observation 1 in [3] with a technique of [5]. The representation $H=\sum I_{m} \delta^{m}$ then follows in $\S 3$ from approximation results for closed derivations given in [1]. Continuity properties of the coefficients are also easily obtained. In $\$ 4$ we consider estimates on the growth of the coefficients $l_{m}$ in terms of certain norms of the linear functionals $f \rightarrow\left(\delta^{m} f\right)(\omega)$. Up to this point it is not necessary to assume that $\delta$ is associated with a flow, or a local flow, but in $\S 5$ we show that if this is the case then our estimates on the $l_{m}$ reproduce those of [3], and provide some improvement in detail.

To conclude this introduction we comment on the notation and assumptions which are to be used throughout the sequel.

First $X$ denotes a locally compact Hausdorff space and $\mathscr{A}=C_{0}(X)$ the space of continuous real-valued functions on $X$ vanishing at infinity, equipped with the supremum norm. Second $\delta: D(\delta) \rightarrow \mathscr{A}$ denotes a closed derivation, $\mathscr{A}_{m}=D\left(\delta^{m}\right)$ the domain of the $m$ th iterate of $\delta$, and $\mathscr{A}_{\infty}=\bigcap_{m=1}^{\infty} \mathscr{A}_{m}$. Note that $\mathscr{A}_{n}$ becomes a Banach space in any of various equivalent norms, for example those used in [3] and [1],

$$
\|f\|_{n}=\sup _{0 \leq m \leq n}\left\|\delta^{m} f\right\|, \quad\|f\|_{\delta, n}=\sum_{m=0}^{n} \frac{1}{m !}\left\|\delta^{m} f\right\|,
$$

and in fact $\|\cdot\|_{\delta, n}$ is an algebra norm. Furthermore $\mathscr{A}_{\infty}$ equipped with either of these families of norms becomes a Fréchet algebra. 
Now suppose $\mathscr{A}_{n}$ is dense in $\mathscr{A}$, for some $1 \leq n \leq \infty$. (If $\delta$ is a generator this condition is automatically satisfied [3], [4].) Since $\delta$ is closed, this ensures that the basic lemmas of $\S 2$ of [1] are applicable, and they will be applied repeatedly in the sequel.

Finally remark that the above framework of real functions and derivations, and of local operators as defined below, is equivalent, by complexification, to the framework of abelian $C^{*}$-algebras and *-derivations, and a similar notion of local operators. Unlike [1], however, it is not possible to assume that $X$ is compact, by passing to the one-point compactification $\tilde{X}$, because a local operator $H$ on $C_{0}(X)$ may have no extension to a local operator on $C(\tilde{X})$.

\section{Locality and continuity}

Let $H: D(H) \rightarrow \mathscr{A}$ denote a linear operator from a subspace $D(H)$ of $\mathscr{A}$ into $\mathscr{A}$. Then $H$ is defined to be local if supp $(H f) \subseteq \operatorname{supp}(f)$ for each $f \in D(H)$. The closed derivation $\delta$ is automatically local [1, lemma 2.4] and, by iteration, $\delta^{m}$ is local. Moreover any operator $H$ of the form

$$
(H f)(\omega)=\sum_{m \geq 0} l_{m}(\omega)\left(\delta^{m} f\right)(\omega)
$$

is local, where defined, for functions $l_{m}: X \rightarrow \mathbb{R}$. Alternatively if $\mathscr{A}_{n}$ is dense in $\mathscr{A}$ and $H$ is a derivation from $\mathscr{A}_{n}$ into $\mathscr{A}$ then $H$ is local by the argument in the second last paragraph of $\S 3$ of [3].

Our immediate aim is to prove continuity of local operators.

Lemma 2.1. Assume $\mathscr{A}_{n}$ is dense in $\mathscr{A}$, for some $1 \leq n \leq \infty$. Let $H: \mathscr{A}_{n} \rightarrow \mathscr{A}$ be a local operator and $F$ the set of points $\omega \in X$ for which the linear functional $f \rightarrow(H f)(\omega)$ is discontinuous on $\mathscr{A}_{n}$. It follows that $F$ is finite and contains no isolated points of $X$.

Proof. If $n=\infty$ then $F$ is finite by the same argument as in observation 1 of [3] (see also [5]). If $n<\infty$ then $\boldsymbol{F}$ is finite by the same argument. In fact in the latter case the proof simplifies because one may take $m=n$ throughout the argument in [3].

Let $\omega$ be an isolated point of $X$. For any function $f \in \mathscr{A}_{n}$ with $f(\omega)=0, \operatorname{supp}(f) \subseteq$ $X \backslash\{\omega\}$, so supp $(H f) \subseteq X \backslash\{\omega\}$ by locality. Thus $(H f)(\omega)=0$. It follows that there is an $l_{0}(\omega) \in \mathbb{R}$ such that

$$
(H f)(\omega)=l_{0}(\omega) f(\omega)
$$

for all $f \in A_{n}$. Hence $\omega \notin F$.

TheOREM 2.2. Assume that $\mathscr{A}_{n}$ is dense in $\mathscr{A}$, for some $1 \leq n \leq \infty$ and let $H: \mathscr{A}_{n} \rightarrow \mathscr{A}$ be a local operator. It follows that $H$ is continuous.

Proof. The proof is similar to that of theorem 2 of [5]. Let $\left\{f_{r}\right\}$ be a sequence in $\mathscr{A}_{n}$ and suppose $f_{r} \rightarrow 0$ in the topology of $\mathscr{A}_{n}$ and $\left\|H f_{r}-g\right\| \rightarrow 0$ for some $g \in \mathscr{A}$. If $\omega \in X \backslash F$, where $F$ is the set of discontinuity introduced in lemma 2.1 , then

$$
g(\omega)=\lim _{r \rightarrow \infty}\left(H f_{r}\right)(\omega)=0 .
$$

Thus $g$ vanishes except possibly at certain points which must both belong to $F$ and be isolated, since $F$ is finite and $g$ is continuous. But no such points exist, by lemma 
2.1. Thus $g=0$, and $H$ has a closed graph. It now follows from the closed graph theorem (for Banach spaces if $n<\infty$, or Fréchet spaces if $n=\infty$ ) that $H$ is continuous.

Remark 2.3. The same proof shows that if $H: \mathscr{A}_{n} \rightarrow \mathscr{A}_{m}$ is a local operator whose range is contained in $\mathscr{A}_{m}$ then $H$ is continuous in the respective topologies of $\mathscr{A}_{n}$ and $\mathscr{A}_{m}$, e.g. if $n, m<+\infty$ then there exists a $C>0$ such that $\|H f\|_{m} \leq C\|f\|_{n}$ for all $f \in \mathscr{A}_{n}$, where $\|\cdot\|_{n}$ and $\|\cdot\|_{m}$ denote the norms used in [3], and redefined in the introduction.

\section{Local operators as differential operators}

Next we establish that each local operator on $\mathscr{A}_{n}$ is a 'differential' operator, i.e. it can be expressed as a finite polynomial in $\delta$.

Theorem 3.1. Assume $\mathscr{A}_{n}$ is dense in $\mathscr{A}$, for some $1 \leq n \leq \infty$ and let $H: \mathscr{A}_{n} \rightarrow \mathscr{A}$ be a local operator. It follows that there exists a finite integer $p \leq n$ and functions $l_{m}: X \rightarrow \mathbb{R}$, $0 \leq m \leq p$, such that

$$
(H f)(\omega)=\sum_{m=0}^{p} l_{m}(\omega)\left(\delta^{m} f\right)(\omega)
$$

for all $f \in \mathscr{A}_{2 n}$ and all $\omega \in X$.

Proof. If $n=\infty$ it follows from theorem 2.2 that $H$ is continuous with respect to $\|\cdot\|_{p}$ for some finite $p$, and if $n<\infty$ we may take $p=n$ and have the same continuity. Thus in both cases there is a finite integer $p \leq n$ and an $a>0$ such that $\|H f\| \leq a\|f\|_{p}$.

Next suppose $\left(\delta^{m} f\right)(\omega)=0$ for $m=0,1, \ldots, p$, for some $\omega \in X$ and $f \in \mathscr{A}_{2 n}$. It follows from proposition 5.2 of [1] that there is a sequence $\left\{f_{j}\right\}$ of functions $f_{j} \in \mathscr{A}_{n}$ such that $f_{j}=0$ in a neighbourhood of $\omega$ and $\left\|f_{j}-f\right\|_{p} \rightarrow 0$ as $j \rightarrow \infty$. (If $n>p$ the statement in [1] does not quite say that $f_{j} \in \mathscr{A}_{n}$ but the proof shows that $f_{j}$ can be chosen to be in $\mathscr{A}_{n}$.) By locality $\left(H f_{j}\right)(\omega)=0$. By continuity $(H f)(\omega)=0$. It follows that there exist real scalars $l_{m}(\omega)$ such that

$$
(H f)(\omega)=\sum_{m=0}^{p} l_{m}(\omega)\left(\delta^{m} f\right)(\omega)
$$

for all $f \in \mathscr{A}_{2 n}$.

Remark 3.2. If $n=\infty$ then $\mathscr{A}_{2 n}=\mathscr{A}_{n}=\mathscr{A}_{\infty}$ and theorem 3.1 describes $H$ on the whole of $\mathscr{A}_{n}$. If $n=1$, so $D(H)=\mathscr{A}_{1}$, then theorem 3.1 only asserts that (*) is valid on $\mathscr{A}_{2}$. But using proposition 5.1 of [1] instead of proposition 5.2 one can conclude that (*) is valid for all $f \in \mathscr{A}_{1}$. If $1<n<\infty$ then (*) is valid for all $f$ in the closure of $\mathscr{A}_{2 n}$ in $\mathscr{A}_{n}$. As on page 321 of [1], if proposition 5.2 can be strengthened to apply to all $f \in \mathscr{A}_{n}$ with $\left(\delta^{m} f\right)(\omega)=0$ for $m=0,1, \ldots, p$, then (*) is valid for all $f \in \mathscr{A}_{n}$.

Remark 3.3. If $\delta$ is associated with a flow $\left\{T_{t}\right\}$ on $X$ as in [3] then $\mathscr{A}_{n}$ is automatically dense in $\mathscr{A}$ and theorem 3.1 applies. But again the conclusion is valid for all $f \in \mathscr{A}_{n}$. This was established in [3] but can also be proved along the above lines by arguing that for each $f \in \mathscr{A}_{n}$ such that $\left(\delta^{m} f\right)(\omega)=0$ for $m=0,1, \ldots, p$, there exists a sequence $f_{j} \in \mathscr{A}_{\infty}$ such that $f_{j}=0$ in a neighbourhood of $\omega$ and $\left\|f_{j}^{\prime}-f\right\|_{p} \rightarrow 0$ as $j \rightarrow \infty$. 
Remark 3.4. Theorem 3.1 shows that the restriction $H^{\prime}$ of $H$ to $\mathscr{A}_{2 n}$ is a local operator with a representation

$$
\left(H^{\prime} f\right)(\omega)=\sum_{m=0}^{p} l_{m}(\omega)\left(\delta^{m} f\right)(\omega), \quad f \in \mathscr{A}_{2 n},
$$

and which is bounded with respect to $\|\cdot\|_{p}$. Under the assumption that $\mathscr{A}_{2 n}$ is dense in $\mathscr{A}$ (which is automatic if $\delta$ generates a flow), the properties of the coefficients $l_{m}$ will be studied in the next section, where, for simplicity, $H$ will be written in place of $H^{\prime}$ and $n$ in place of $2 n$. Similarly, by remark 3.2, the results of $\S 4$ apply to any local operator defined on $\mathscr{A}_{1}$.

\section{Continuity and growth of coefficients}

In this section we consider a local operator $H: \mathscr{A}_{n} \rightarrow \mathscr{A}$, where $1 \leq n \leq \infty$ and $\mathscr{A}_{n}$ is assumed to be dense in $\mathscr{A}$, which is given by a representation

$$
(H f)(\omega)=\sum_{m=0}^{p} l_{m}(\omega)\left(\delta^{m} f\right)(\omega), \quad f \in \mathscr{A}_{n},
$$

where $1 \leq p \leq n$ is a finite integer. We assume $H$ is bounded with respect to $\|\cdot\|_{p}$. (If $n=p$ the continuity is automatic by the closed graph theorem.) Our aim is to prove uniqueness and continuity of the coefficient functions $l_{m}$ and to derive bounds on their growth.

First introduce the 'fixed point' set

$$
X_{0}=\left\{\omega \in X ;(\delta f)(\omega)=0 \text { for all } f \in \mathscr{A}_{n}\right\} .
$$

Note that if $\delta$ is the generator of a flow $\left\{T_{t}\right\}$ on $X$, as in [3], then

$$
X_{0}=\left\{\omega \in X ; T_{1} \omega=\omega \text { for all } t \in \mathbb{R}\right\} \text {. }
$$

LeMmA 4.1. For $1 \leq k \leq n$ and $\omega \in X \backslash X_{0}$ there exists an $f \in \mathscr{A}_{n}$ such that $\left(\delta^{m} f\right)(\omega)=0$ for $0 \leq m<k$ but $\left(\delta^{k} f\right)(\omega)=1$.

Proof. By assumption there exists a $g \in \mathscr{A}_{n}$ with $(\delta g)(\omega)=1$. But by addition of a function which is constant near $\omega$ we may arrange that $g(\omega)=1$. Then

$$
\lim _{q \rightarrow \infty} \frac{\left(\delta^{k} g^{q}\right)(\omega)}{q^{k}}=1,
$$

but

$$
\lim _{q \rightarrow \infty} \frac{\left(\delta^{m} g^{q}\right)(\omega)}{q^{k}}=0
$$

for $0 \leq m<k$. Thus the linear functional $f \rightarrow\left(\delta^{k} f\right)(\omega)$ on $\mathscr{A}_{n}$ is not a linear combination of functionals $f \rightarrow\left(\delta^{m} f\right)(\omega), 0 \leq m<k$. Therefore there exists an $f \in \mathscr{A}_{n}$ with the required properties.

Corollary 4.2. If $\omega \in X \backslash X_{0}$ and $0 \leq k \leq n$ then there exists an $f_{k} \in \mathscr{A}_{n}$ such that $\left(\delta^{k} f_{k}\right)(\omega)=1$ but $\left(\delta^{m} f_{k}\right)(\omega)=0$ for $0 \leq m \leq n, m \neq k$.

Proof. First choose $g_{0} \in \mathscr{A}_{n}$ such that $g_{0}(\omega)=1$. Next by lemma 4.1 there exists for $1 \leq j \leq n$ a $g_{j} \in \mathscr{A}_{n}$ such that $\left(\delta^{j} g_{j}\right)(\omega)=1$, but $\left(\delta^{i} g_{j}\right)(\omega)=0$ for $0 \leq i<j$. It follows immediately that one can construct linear combinations $f_{k}$ of $g_{0}, g_{1}, \ldots, g_{n}$ with the desired properties. 
Proposition 4.3. The function $l_{0}$ is uniquely determined by $H$ and continuous on $X$. For $1 \leq k \leq p$ the function $l_{k}$ is uniquely determined by $H$ and continuous on $X \backslash X_{0}$. Proof. Let $\omega \in X$ and choose $f_{0} \in \mathscr{A}_{n}$ equal to one near $\omega$. Then $l_{0}=H f_{0}$ in the same open neighbourhood of $\omega$. Thus $l_{0}$ is uniquely determined and continuous at $\omega$.

If $\omega \in X \backslash X_{0}$ we can, by corollary 4.2 , choose a function $f_{k} \in \mathscr{A}_{n}$ such that $\left(\delta^{k} f_{k}\right)(\omega)=1$ but $\left(\delta^{m} f_{k}\right)(\omega)=0$ for $0 \leq m \leq n, m \neq k$. Then $l_{k}(\omega)=\left(H f_{k}\right)(\omega)$ and hence $l_{k}$ is uniquely determined by $H$ on $X \backslash X_{0}$. Furthermore the linear equations

$$
\sum_{i=1}^{p} l_{i}\left(\omega^{\prime}\right)\left(\delta^{i} f_{j}\right)\left(\omega^{\prime}\right)=\left(H f_{j}\right)\left(\omega^{\prime}\right)-l_{0}\left(\omega^{\prime}\right) f_{j}\left(\omega^{\prime}\right)
$$

for $l_{i}\left(\omega^{\prime}\right), 1 \leq i \leq n$, have continuous coefficients and are non-singular at $\omega$. Thus the $l_{i}$ are continuous at $\omega$.

Next we consider the growth properties of the coefficients $l_{m}$. By assumption the local operator $H=\sum_{m=0}^{p} l_{m} \delta^{m}$ from $\mathscr{A}_{n}$ into $\mathscr{A}$ is bounded with respect to $\|\cdot\|_{p}$ on $\mathscr{A}_{n}$ so there is a constant $a$ such that

$$
|(H f)(\omega)| \leq a\|f\|_{p}
$$

for all $f \in \mathscr{A}_{n}$ and $\omega \in X$.

Lemma 4.4. For $\omega \in X$ and $f \in \mathscr{A}_{n}$

$$
\left|l_{p}(\omega)(\delta f)(\omega)^{p}\right| \leq a\|\delta f\|^{p}
$$

Proof. Given $\varepsilon>0$ let $g: \mathbb{R} \rightarrow \mathbb{R}$ be a $C^{\infty}$-function with the properties

$$
\begin{aligned}
& g(0)=0 ; \\
& g^{(p)}(f(\omega))=1 ; \\
& \left|g^{(p)}(t)\right| \leq 1, \quad t \in \mathbb{R} ; \\
& g^{(m)}(f(\omega))=0, \quad 0 \leq m<p ; \\
& \left|g^{(m)}(t)\right|<\varepsilon, \quad 0 \leq m<p, t \in \mathbb{R} .
\end{aligned}
$$

Then $g \circ f \in \mathscr{A}_{n}$ and

$$
\begin{gathered}
\left\|\delta^{m}(g \circ f)\right\| \leq N_{m} \varepsilon\|f\|_{p}^{m}, \quad 0 \leq m<p, \\
\left\|\delta^{p}(g \circ f)-\left(g^{(p)} \circ f\right)(\delta f)^{p}\right\| \leq N_{p} \varepsilon\|f\|_{p}^{p},
\end{gathered}
$$

for certain integers $N_{m}$ independent of $\varepsilon$, and of $\delta, f, g$ (see, for example, lemma 2.2 of $[1])$. Hence

$$
\begin{aligned}
&\left|l_{p}(\omega)(\delta f)(\omega)^{p}\right| \leq|H(g \circ f)(\omega)|+\left|\sum_{m=0}^{p-1} l_{m}(\omega)\left(\delta^{m}(g \circ f)\right)(\omega)\right| \\
&+\left|l_{p}(\omega)\right|\left\|\delta^{p}(g \circ f)-\left(g^{(p)} \circ f\right)(\delta f)^{p}\right\| \\
& \leq a\left\|\delta^{p}(g \circ f)\right\|+\sum_{m=0}^{p-1}\left(a+\left|l_{m}(\omega)\right|\right)\left\|\delta^{m}(g \circ f)\right\| \\
&+\left|l_{p}(\omega)\right|\left\|\delta^{p}(g \circ f)-\left(g^{(p)} \circ f\right)(\delta f)^{p}\right\| \\
& \leq a\|\delta f\|^{p}+\varepsilon \sum_{m=0}^{p}\left(a+\left|l_{m}(\omega)\right|\right) N_{m}\|f\|_{p}^{m}
\end{aligned}
$$


Taking the limit as $\varepsilon \rightarrow 0$ one then obtains

$$
\left|l_{p}(\omega)(\delta f)(\omega)^{p}\right| \leq a\|\delta f\|^{p} .
$$

Proposition 4.5. If $|(H f)(\omega)| \leq a\|f\|_{p}$ for all $f \in \mathscr{A}_{n}$ and $\omega \in X$ then $\left|l_{p}(\omega)\right| \leq a$ for all $\omega \in X \backslash X_{0}$.

Proof. It follows from lemma 4.4 that $\left|l_{p}(\omega)(\delta f)(\omega)^{p}\right| \leq a\|\delta f\|^{p}$ for all $\omega \in X$ and $f \in \mathscr{A}_{n}$. Now suppose $\left|l_{p}\left(\omega_{0}\right)\right|>a$ for some $\omega_{0} \in X \backslash X_{0}$. Since $l_{p}$ is continuous on $X \backslash X_{0}$, by proposition 4.3 , there is an open subset $U$ of $X \backslash X_{0}$ such that $\left|l_{p}(\omega)\right|>a$ for all $\omega \in U$. Next let $f \in \mathscr{A}_{n}$ be a function such that $\operatorname{supp}(f) \subseteq U$ and $\delta f \neq 0$. Such functions exist by functional calculus of the domain of $\delta$. Now choose $\omega_{1} \in X$ such that $\left|(\delta f)\left(\omega_{1}\right)\right|=\|\delta f\|$. Since supp $(\delta f) \subseteq U$ it follows that $\omega_{1} \in U$ and

$$
\left|l_{p}\left(\omega_{1}\right)\right|\|\delta f\|^{p}=\left|l_{p}\left(\omega_{1}\right)(\delta f)\left(\omega_{1}\right)^{p}\right| \leq a\|\delta f\|^{p} .
$$

But this is inconsistent with the fact that $\left|l_{p}\left(\omega_{1}\right)\right|>a$. Thus $\left|l_{p}(\omega)\right| \leq a$ for all $\omega \in$ $X \backslash X_{0}$.

Remark 4.6. A calculation similar to that in lemma 4.4 and an inductive argument show that if $H=\sum_{m=0}^{p} l_{m} \delta^{m}$ is bounded with respect to $\|\cdot\|_{q}$ on $\mathscr{A}_{n}$ where $q \leq p \leq n$ then $l_{m}=0$ on $X \backslash X_{0}$ for $m>q$, and $l_{q}$ is bounded on $X \backslash X_{0}$ by proposition 4.5.

The coefficients $l_{m}$ with $0 \leq m<p$ are not as easy to estimate as $l_{p}$. Let

$$
d_{m}(\omega)=\sup \left\{\left|\left(\delta^{m} f\right)(\omega)\right| ; f \in \mathscr{A}_{n},\|f\|_{p} \leq 1 \text { and }\left(\delta^{q} f\right)(\omega)=0 \text { for } 0 \leq q \leq p, q \neq m\right\} \text {. }
$$

Then the bounds $|(H f)(\omega)| \leq a\|f\|_{p}$ immediately imply $\left|l_{m}(\omega)\right| d_{m}(\omega) \leq a$. But for any $\omega \in X$ one can readily deduce that $d_{0}(\omega)>0$ by choosing an $f \in \mathscr{A}_{n}$ which is constant in an open neighbourhood of $\omega$. Moreover if $\omega \in X \backslash X_{0}$ and $1 \leq m \leq p$ then $d_{m}(\omega)>0$ by corollary 4.2. Therefore under these circumstances one has the bounds

$$
\left|l_{m}(\omega)\right| \leq a / d_{m}(\omega) .
$$

Unfortunately the $d_{m}$ are difficult to calculate in general because their definition involves conditions on all derivatives $\delta^{q} f$ with $0 \leq q \leq p$, of functions $f \in \mathscr{A}_{n}$. In the next section we demonstrate that the $d_{m}$ can be efficiently estimated if $\delta$ is associated with a flow $\left\{T_{t}\right\}$ on $X$ but to conclude this section we derive alternative bounds for the $l_{m}$ in terms of the somewhat simpler functions

$$
\begin{aligned}
& C_{m}(\omega)=\sup \left\{\left|\left(\delta^{m} f\right)(\omega)\right| ; f \in \mathscr{A}_{n},\|f\|_{p} \leq 1\right\} \\
& C_{m}^{\prime}(\omega)=\sup \left\{\left|\left(\delta^{m} f\right)(\omega)\right| ; f \in \mathscr{A}_{n},\|f\|_{p} \leq 1 \text { and }\left(\delta^{q} f\right)(\omega)=0 \text { for } 0 \leq q \leq m-1\right\} .
\end{aligned}
$$

The following inequalities simplify the estimation of $C_{m}$ and $C_{m}^{\prime}$.

LEMMA 4.7. If $\omega \in X$ and $1 \leq m \leq p$ then

$$
\begin{aligned}
\left(2^{p}+1\right) C_{1}^{\prime}(\omega) & \geq C_{1}(\omega) C_{0}(\omega), \\
m^{p} C_{m}^{\prime}(\omega) & \geq m ! C_{1}^{\prime}(\omega)^{m} .
\end{aligned}
$$

Proof. Let $f_{1}, f_{2} \in \mathscr{A}_{n}$ with $\left\|f_{1}\right\|_{p} \leq 1,\left\|f_{2}\right\|_{p} \leq 1$. Set

$$
f=f_{1} f_{2}-f_{1}(\omega) f_{2} \text {. }
$$

Then $\|f\|_{p} \leq 2^{p}+1, f(\omega)=0$, and $(\delta f)(\omega)=\left(\delta f_{1}\right)(\omega) f_{2}(\omega)$. Hence

$$
\left|\left(\delta f_{1}\right)(\omega) f_{2}(\omega)\right|=|(\delta f)(\omega)| \leq\left(2^{p}+1\right) C_{1}^{\prime}(\omega) .
$$


Taking the supremum over all possible $f_{1}, f_{2}$ gives the first inequality.

Next suppose $h \in \mathscr{A}_{n}, h(\omega)=0$, and $\|h\|_{p} \leq 1$. Then

$$
\left(\delta^{q} h^{m}\right)(\omega)=0 \quad \text { for } 0 \leq q<m \text { and } \quad\left(\delta^{m} h^{m}\right)(\omega)=m !(\delta h)(\omega)^{m} .
$$

Moreover $\left\|h^{m}\right\|_{p} \leq m^{p}$. Hence

$$
m !|(\delta h)(\omega)|^{m} \leq m^{p} C_{m}^{\prime}(\omega) .
$$

Taking the supremum over all possible $h$ gives the second inequality.

Proposition 4.8. Let $Y$ be a subset of $X$ and suppose there exist constants $\beta_{m} \geq 0$ such that $\beta_{m} C_{m}^{\prime}(\omega) \geq C_{m}(\omega)$ for all $\omega \in Y$ and $0 \leq m \leq p$. Then

$$
\left|l_{m}(\omega)\right| C_{m}(\omega) \leq a \beta_{m} \prod_{q=m+1}^{p}\left(1+\beta_{q}\right)
$$

for all $\omega \in Y$ and $0 \leq m \leq p$.

Proof. Firstly $C_{p}^{\prime}=d_{p}$ so

$$
\left|l_{p}(\omega)\right| C_{p}(\omega) \leq \beta_{p}\left|l_{p}(\omega)\right| d_{p}(\omega) \leq a \beta_{p}
$$

Next suppose the assertion is true for all $m=k+1, k+2, \ldots, p$ where $0 \leq k \leq p$. Take $f \in \mathscr{A}_{n}$ with $\left(\delta^{a} f\right)(\omega)=0$ for $0 \leq q<k,\|f\|_{p} \leq 1$. Then

and hence

$$
\left|\sum_{q=k}^{p} l_{q}(\omega)\left(\delta^{q} f\right)(\omega)\right| \leq a
$$

$$
\begin{aligned}
\left|l_{k}(\omega)\left(\delta^{k} f\right)(\omega)\right| & \leq a+\sum_{q=k+1}^{p}\left|l_{q}(\omega)\left(\delta^{q} f\right)(\omega)\right| \\
& \leq a+\sum_{q=k+1}^{p}\left|l_{q}(\omega)\right| C_{q}(\omega) \\
& \leq a+\sum_{q=k+1}^{p} a \beta_{q} \prod_{r=q+1}^{p}\left(1+\beta_{r}\right) \\
& =a \prod_{q=k+1}^{p}\left(1+\beta_{q}\right) .
\end{aligned}
$$

Taking the supremum over all possible $f$ gives

$$
\left|l_{k}(\omega)\right| C_{k}^{\prime}(\omega) \leq a \prod_{q=k+1}^{p}\left(1+\beta_{q}\right)
$$

and consequently

$$
\left|l_{k}(\omega)\right| C_{k}(\omega) \leq a \beta_{k} \prod_{q=k+1}^{p}\left(1+\beta_{q}\right)
$$

Thus the assertion is true for all $k=0,1, \ldots, p$ by induction.

Combining these results one obtains bounds on the $l_{m}$ in terms of the relatively simple functions $C_{m}$ on certain subsets of $X$ which are in turn determined by $C_{0}$ and $C_{1}$.

Corollary 4.9. Let $\gamma_{0}, \gamma_{1}>0$ and define $Y$ by

$$
Y=\left\{\omega \in X ; C_{0}(\omega) \geq \gamma_{0}, C_{1}(\omega) \geq \gamma_{1}\right\} .
$$


It follows that there exist constants $\alpha_{m}$ such that

$$
\left|l_{m}(\omega)\right| C_{m}(\omega) \leq \alpha_{m}
$$

for all $\omega \in Y$ and $m=0,1, \ldots, p$.

Proof. Clearly $C_{m}(\omega) \leq 1$. But by lemma 4.7

$$
\begin{aligned}
m^{p} C_{m}^{\prime}(\omega) & \geq m !\left(C_{1}(\omega) C_{0}(\omega)\right)^{m} /\left(2^{p}+1\right)^{m} \\
& \geq m !\left(\gamma_{0} \gamma_{1} /\left(2^{p}+1\right)\right)^{m}
\end{aligned}
$$

for all $\omega \in Y$ and $m=0,1, \ldots, p$. Thus proposition 4.8 is applicable with $\beta_{m}=$ $\left(m^{p} / m !\right)\left(\left(2^{p}+1\right) / \gamma_{0} \gamma_{1}\right)^{m}$.

\section{Flows}

In this section we assume $\delta$ is associated with a flow $\left\{T_{t}\right\}$ on $X$, as in [3]. Thus $\delta$ is the generator of the one-parameter group $\tau$ of *-automorphisms of $C_{0}(X)$ given by $\tau_{t} f=f \circ T_{t}$. It follows automatically that $\mathscr{A}_{n}$ is dense in $\mathscr{A}$ for all $1 \leq n \leq \infty$, and the results of the preceding sections apply. Moreover $X_{0}$ is equal to the fixed point set of $T$,

$$
X_{0}=\left\{\omega \in X ; T_{t} \omega=\omega \text { for all } t \in \mathbb{R}\right\} .
$$

Thus if $H: \mathscr{A}_{n} \rightarrow \mathscr{A}$ is a local operator there is a finite integer $1 \leq p \leq n$, an $a>0$ such that $\|H f\| \leq a\|f\|_{p}$ for all $f \in \mathscr{A}_{n}$, and functions $l_{m}: X \rightarrow \mathbb{R}, 0 \leq m \leq p$, such that

for all $f \in \mathscr{A}_{n}$ and $\omega \in X$.

$$
(H f)(\omega)=\sum_{m=0}^{p} l_{m}(\omega)\left(\delta^{m} f\right)(\omega),
$$

The function $l_{0}$ is uniquely determined by $H$ on $X$, continuous on $X$, and satisfies

$$
\left|l_{0}(\omega)\right| \leq a / d_{0}(\omega), \quad \omega \in X .
$$

The other coefficients $l_{m}, 1 \leq m \leq p$, are uniquely determined by $H$ on $X \backslash X_{0}$, continuous on $X \backslash X_{0}$, and satisfy

$$
\left|l_{m}(\omega)\right| \leq a / d_{m}(\omega), \quad \omega \in X \backslash X_{0} .
$$

Our aim is to estimate these bounds in a more explicit fashion, and to reproduce the polynomial bounds of [3].

First recall from $\$ 4$ that

$$
d_{m}(\omega)=\sup \left\{\left|\left(\delta^{m} f\right)(\omega)\right| ; f \in \mathscr{A}_{n},\|f\|_{p} \leq 1 \text { and }\left(\delta^{q} f\right)(\omega)=0 \text { for } 0 \leq q \leq p, q \neq m\right\} .
$$

OBSERVATION 5.1. It follows that $d_{0}(\omega)=1$ for all $\omega \in X$.

Proof. Let $g: \mathbb{R} \rightarrow[0,1]$ be a $C^{\infty}$-function with compact support $K$ such that

$$
\begin{aligned}
& \int d t g(t)=1, \\
& \int d t\left|g^{(m)}(t)\right| \leq 1, \quad 1 \leq m \leq p .
\end{aligned}
$$

Given $\omega \in X$ let $h \in \mathscr{A}$ be such that $h\left(T_{t} \omega\right)=1$ for $t \in K$ and $\|h\|=1$. Define

$$
f=\int d t g(t) h \circ T_{t} .
$$


Then $f \in \mathscr{A}_{\infty}, f(\omega)=1$, and $\left(\delta^{q} f\right)(\omega)=0$ for $q \geq 1$. Moreover $\|f\|_{p}=1$. Therefore $d_{0}(\omega)=1$.

The values of $d_{m}(\omega)$ for $m \geq 1$ are more difficult to calculate and depend critically on the behaviour of $\omega$ under the flow $T$. A simplifying feature is that they are given by the corresponding coefficients for translations on the line or rotations on the circle.

Observation 5.2. Let $S(\omega)$ denote the stabilizer subgroup of $\omega \in X \backslash X_{0}$, i.e. $S(\omega)=\left\{t \in \mathbb{R} ; T_{t} \omega=\omega\right\}$. It follows that

$$
\begin{gathered}
d_{m}(\omega)=\sup \left\{\left|F^{(m)}(0)\right| ; F \in C_{c}^{\infty}(\mathbb{R} / S(\omega)),\|F\|_{p} \leq 1, \text { and } F^{(q)}(0)=0\right. \\
\text { for } 0 \leq q \leq p, q \neq m\}
\end{gathered}
$$

where $C_{c}^{\infty}(\mathbb{R} / S(\omega))$ denotes the space of infinitely differentiable functions of compact support on the quotient group $\mathbb{R} / S(\omega)$ and

$$
\|F\|_{p}=\sup _{0 \leq k \leq p}\left\|F^{(k)}\right\| .
$$

Remark 5.3. Since $\omega \in X \backslash X_{0}$ is not a fixed point of $T$ there are two possibilities. Either $S(\omega)=\{0\}$, i.e. the orbit of $\omega$ under $T$ is aperiodic, in which case $\mathbb{R} / S(\omega)=\mathbb{R}$. Then the value of $d_{m}(\omega)$ corresponds to that associated with translations acting on $C_{0}(\mathbb{R})$. Or $S(\omega)=\rho \mathbb{Z}$, i.e. the orbit of $\omega$ is periodic with period $\rho=\rho(\omega)$, and $\mathbb{R} / S(\omega)$ is a circle group. Then the value of $d_{m}(\omega)$ corresponds to that associated with rotations acting on $C(\mathbb{R} / \rho \mathbb{Z})$.

Proof. Take $f \in \mathscr{A}_{n}$ with $\|f\|_{p} \leq 1$ and $\left(\delta^{q} f\right)(\omega)=0$ for $0 \leq q \leq p, q \neq m$. There is a sequence $f_{k} \in \mathscr{A}_{\infty}$ such that $\left\|f_{k}-f\right\|_{n} \rightarrow 0$. By adding linear combinations of certain fixed functions, one may assume that $\left(\delta^{q} f_{k}\right)(\omega)=0$ for $0 \leq q \leq p, q \neq m$. Next let $g$ be a fixed function in $C_{c}^{\infty}(\mathbb{R} / S(\omega))$ such that $g(t)=1$ near $t=0$ and define $F_{k}$ by $F_{k}(t)=g(t / k) f_{k}\left(T_{t} \omega\right)$. Then $F_{k} \in C_{c}^{\infty}(\mathbb{R} / S(\omega))$, lim sup $\left\|F_{k}\right\|_{p} \leq 1$, and $F_{k}^{(m)}(0) \rightarrow$ $\left(\delta^{m} f\right)(\omega)$. Consequently

$$
\begin{gathered}
d_{m}(\omega) \leq \sup \left\{\left|F^{(m)}(0)\right| ; F \in C_{c}^{\infty}(\mathbb{R} / S(\omega)),\|F\|_{p} \leq 1 \text { and } F^{(q)}(0)=0\right. \\
\text { for } 0 \leq q \leq p, q \neq m\} .
\end{gathered}
$$

Conversely if $F \in C_{c}^{\infty}(\mathbb{R} / S(\omega))$ then $F=\sum_{i=1}^{N} G_{i} * H_{i}$ for some functions $G_{i}$, $H_{i} \in C_{c}^{\infty}(\mathbb{R} / S(\omega)$ ) by [7]. (In fact if $S(\omega) \neq\{0\}$ then one may take $N=1$ since the Fourier coefficients tend to zero faster than any power; if $S(\omega)=\{0\}$ one may take $N=2$ by [7].) Next let $h_{i}$ be a function in $C_{0}(X)$ such that $h_{i}\left(T_{t} \omega\right)=H_{i}(t)$ for $t \in[-1,1]+\operatorname{supp}(F)-\operatorname{supp}\left(G_{i}\right)$ and define $f$ by

$$
f\left(\omega^{\prime}\right)=\sum_{i=1}^{N} \int d s G_{i}(s) h_{i}\left(T_{-s} \omega^{\prime}\right) .
$$

It follows that $f \in \mathscr{A}_{\infty}$ and $f\left(T_{t} \omega\right)=F(t)$ if $t \in[-1,1]+\operatorname{supp}(F)$. Consequently, by theorem 2.1 of [3], there exists for any $\varepsilon>0$ an $f_{1} \in \mathscr{A}_{\infty}$ such that $\left\|f_{1}\right\|_{p} \leq(1+\varepsilon)\|F\|_{p}$ and $f_{1}\left(T_{t} \omega\right)=F(t)$ near $t=0$. If $\|F\|_{p}<1$ and $F^{(q)}(0)=0$ for $0 \leq q \leq p, q \neq m$, then $\left(\delta^{q} f_{1}\right)(\omega)=0$ for $0 \leq q \leq p, q \neq m$, and, by choosing $\varepsilon$ sufficiently small, one may arrange that $\left\|f_{1}\right\|_{p}<1$. Thus

This completes the proof.

$$
d_{m}(\omega) \geq\left|\left(\delta^{m} f_{1}\right)(\omega)\right|=\left|F^{(m)}(0)\right| .
$$


Note that in the periodic case, i.e. $S(\omega) \neq\{0\}$, one may alternatively prove this second inequality by showing that for any trigonometric monomial $F$ there exists an $f \in \mathscr{A}_{n}$ such that $f\left(T_{t} \omega\right)=F(t)$.

Observation 5.2 shows that $d_{m}(\omega)$ depends only on $\rho(\omega)$ and therefore we shall write $d_{m}(\rho)$ for $d_{m}(\omega)$ where $\rho=\rho(\omega)$, with the convention that $\rho(\omega)=\infty$ if $\omega$ is aperiodic.

OBSERVATION 5.4. It follows that $d_{p}(\rho)=1$ for all $0<\rho \leq \infty$.

Proof. For any $g \in C_{c}^{\infty}(\mathbb{R})$ let $g_{k}(t)=k^{-p} g(k t)$. For large $k$ the function $g_{k}$ may be regarded as a function of period $\rho$. Furthermore $\left\|g_{k}\right\|_{p} \rightarrow\left\|g^{(p)}\right\|$ and $g_{k}^{(p)}(0)=g^{(p)}(0)$. Hence

$$
\begin{array}{rlrl}
1 \geq d_{p}(\rho) & \geq \sup \left\{\left|g^{(p)}(0)\right|\right. & ; g \in C_{c}^{\infty}(\mathbb{R}),\left\|g^{(p)}\right\| \leq 1, \text { and } \\
& =1 & \left.g^{(q)}(0)=0 \text { for } 0 \leq q<p\right\} \\
&
\end{array}
$$

For $0<m<p$ calculation of the exact value of $d_{m}(p)$ is neither straightforward nor particularly instructive since it depends on the arbitrary choice of the norm $\|\cdot\|_{p}$. But it is useful to know the asymptotic behaviour of $d_{m}$.

OBSERVATION 5.5. If $0<\rho \leq 2$ then $d_{m}(\rho)=\rho^{p-m} d_{m}(1)$ and if $2 \leq \rho \leq \infty$ then $1 \geq d_{m}(\rho) \geq d_{m}(1)$.

Proof. Assume $0<\rho \leq 2$. If $F \in C^{\infty}(\mathbb{R} / \rho \mathbb{Z}),\left\|F^{(p)}\right\| \leq 1$, and $F(0)=0$, then it follows that $\|F\|_{p} \leq 1$. Hence

$$
\begin{array}{r}
d_{m}(\rho)=\sup \left\{\left|F^{(m)}(0)\right| ; F \in C^{\infty}(\mathbb{R} / \rho \mathbb{Z}),\left\|F^{(p)}\right\| \leq 1,\right. \text { and } \\
\left.F^{(q)}(0)=0 \text { for } 0 \leq q \leq p, q \neq m\right\} .
\end{array}
$$

The change of variable $t \rightarrow t / \rho$ immediately gives $d_{m}(\rho)=\rho^{p-m} d_{m}(1)$.

Since any function of period $\rho / 2$ may also be regarded as a function of period $\rho$ one has $d_{m}(\rho) \geq d_{m}(\rho / 2)$. Therefore if $\rho \geq 2$ one has by iteration

$$
\begin{aligned}
d_{m}(\rho) & \geq \inf \left\{d_{m}\left(\rho^{\prime}\right) ; 1 \leq \rho^{\prime} \leq 2\right\} \\
& =\inf \left\{\left(\rho^{\prime}\right)^{p-m} d_{m}(1) ; 1 \leq \rho^{\prime} \leq 2\right\}=d_{m}(1),
\end{aligned}
$$

where the first equality follows from the first part. Finally it is evident that $d_{m}(\rho) \leq 1$ for all $\rho$.

These observations now give the following bounds on the coefficients $l_{m}$ of the local operator $H$.

(1) $\left|l_{0}(\omega)\right| \leq a$, for $\omega \in X$;

(2) $\left|l_{p}(\omega)\right| \leq a$, for $\omega \in X \backslash X_{0}$;

(3) $\left|l_{m}(\omega)\right| \leq a / d_{m}(\infty)$, for $m=1,2, \ldots, p-1$, if $S(\omega)=\{0\}$;

(4) $\left|l_{m}(\omega)\right| \leq\left(a / d_{m}(1)\right)\left(1+(1 / \rho)^{p-m}\right)$, for $m=1,2, \ldots, p-1$, if $S(\omega)=\rho \mathbb{Z}$ where $\rho>0$.

This reproduces the polynomial boundedness results discovered in [3] but with more specific estimates. 
A number of these observations immediately apply to multi-dimensional flows.

Adopting the multi-index notation of [3] it follows as in the earlier sections that a local operator $H: \mathscr{A}_{n} \rightarrow \mathscr{A}$ satisfies a bound $\|H f\| \leq a\|f\|_{p}$ for all $f \in \mathscr{A}_{n}$, and can be expressed as a polynomial $H=\sum_{|\alpha| \leqslant p} l_{\alpha} \delta^{\alpha}$ in the generators of the corresponding group $\tau$. Observations 5.1 and 5.2 readily extend to this case. Thus for $\omega \in X$ $\left|l_{0}(\omega)\right| \leq a, d_{\alpha}(\omega)\left|l_{\alpha}(\omega)\right| \leq a$, for $|\alpha|=1,2, \ldots, p$, and

$$
\begin{gathered}
d_{\alpha}(\omega)=\sup \left\{\left|\left(D^{\alpha} F\right)(0)\right| ; F \in C_{c}^{\infty}\left(\mathbb{R}^{\nu} / S(\omega)\right),\|F\|_{p} \leq 1\right. \\
\text { and } \left.\left(D^{\beta} F\right)(0)=0 \text { for }|\beta| \leq p, \beta \neq \alpha\right\} .
\end{gathered}
$$

But now there is a variety of possibilities for the stabilizer subgroups $S(\omega)$. The simplest case is if $T$ acts freely, i.e. $S(\omega)=\{0\}$ for all $\omega \in X$. Then it follows from the above identification that all the coefficients $l_{\alpha}$ are bounded. This reproduces theorem 1.1A of [3].More generally if $S(\omega)$ is discrete and

$$
\rho=\inf \{|t|: t \in S(\omega), t \neq 0\},
$$

then the coefficients $l_{\alpha}(\omega)$ are uniquely determined, and

$$
\left|l_{\alpha}(\omega)\right| \leq a c_{\alpha}\left(1+(1 / \rho)^{p-|\alpha|}\right)
$$

for some constant $c_{\alpha}$ depending only on $\rho$ and $\alpha$.

The above methods also allow one to obtain estimates on the coefficients of local operators associated with local flows (see [3, § 5]). The one-dimensional case is again the simplest. If the local flow is the restriction of the global flow $T$ to some open subset then one defines the period $\rho=\rho(\omega)$ of a point $\omega$ as the largest value of $K>0$ such that $T_{t} \omega$ is defined for all $t \in\langle-K / 2, K / 2\rangle$ and $T_{\langle-K / 2, K / 2\rangle} \omega$ is an injective image of the interval $\langle-K / 2, K / 2\rangle$. Then by modification of the above arguments one finds bounds

$$
\left|l_{m}(\omega)\right| \leq a / \hat{d}_{m}(\rho)
$$

where $\hat{d}_{m}$ is given by

$$
\begin{gathered}
\hat{d}_{m}(\rho)=\sup \left\{\left|F^{(m)}(0)\right| ; F \in C_{c}^{\infty}(-\rho / 2, \rho / 2),\|F\|_{p} \leq 1,\right. \text { and } \\
\left.F^{(q)}(0)=0 \text { for } 0 \leq q \leq p, q \neq m\right\}
\end{gathered}
$$

for all $0<\rho \leq \infty$. In this case the functions $\rho \rightarrow \hat{d}_{m}(\rho)$ are monotonically increasing and

$$
\hat{d}_{m}(\rho)=\rho^{p-m} \hat{d}_{m}(1)
$$

for $0 \leq \rho \leq 1$ by the argument used to prove observation 5.5. Therefore

$$
\left|l_{m}(\omega)\right| \leq\left(a / \hat{d}_{m}(1)\right)\left(1+(1 / \rho)^{p-m}\right)
$$

Moreover $\hat{d}_{0}(\omega)=1=\hat{d}_{p}(\omega)$ as above.

Example 5.6. The operator of differentiation $\delta=d / d x$ on $C_{0}(0, \infty)$ is the generator of the local flow obtained by restricting translations to the half-axis. Then for $x \in(0, \infty)$ one has $\rho(x)=2 x$ and the coefficients $l_{m}$ of a local operator $H$ satisfy the bounds $\left|l_{m}(x)\right| \leq C_{m, p}\left(1+x^{-p+m}\right)$ for some $C_{m, p} \geq 0$.

Finally we remark that boundedness of the coefficient $l_{0}$ is a special feature of the generator case. For non-generators, $l_{0}$ may be unbounded. 
Example 5.7. Let $X=(0,1]$. Define $\delta$ and $H$ by

$$
\begin{gathered}
D(\delta)=\left\{f \in C^{1}(0,1] ; f^{\prime} \in C_{0}(0,1]\right\}, \\
\delta f=f^{\prime}, \quad D(H)=D(\delta), \quad(H f)(t)=t^{-1} f(t) .
\end{gathered}
$$

Then $H$ is a local operator from $\mathscr{A}_{1}$ into $\mathscr{A}$ but the coefficient $l_{0}(t)=t^{-1}$ is unbounded. Further if one defines $H_{1}: \mathscr{A}_{2} \rightarrow \mathscr{A}$ by

$$
\left(H_{1} f\right)(t)=t^{-1} f^{\prime}(t)
$$

then $H_{1}$ is a derivation but the coefficient $l_{1}(t)=t^{-1}$ is unbounded. (This shows that the word 'bounded' must be deleted from theorem 5 of [2] when it is applied to derivations with domain $\mathscr{A}_{n}$ for $n>1$.)

Acknowledgements. This work was carried out mainly whilst the authors were visiting the University of Warwick. We are grateful to Dai Evans for his hospitality, to Ola Bratteli and Trond Digernes for helpful discussions, and to the Science and Engineering Research Council for their financial support of the second author.

\section{REFERENCES}

[1] C. J. K. Batty. Derivations on compact spaces. Proc. London Math. Soc. 42 (1981), 299-330.

[2] C. J. K. Batty. Derivations of abelian $C^{*}$-algebras. Proc. Symp. Pure Math. 38 (1982) part 2, 333-338.

[3] O. Bratteli, G. A. Elliott \& D. W. Robinson. The characterization of differential operators by locality: classical flows. Trondheim preprint (1984).

[4] E. B. Davies. One-parameter Semigroups. Academic Press, (1980).

[5] B. E. Johnson. Continuity of derivations on commutative algebras. Amer. J. Math. 91 (1969), 1-10.

[6] J. Peetre. Une caractérisation abstraite des opérateurs différentiels. Math. Scand. 7 (1959), 211-218; and Rectification à l'article précédent. Math. Scand. 8 (1960), 116-120.

[7] L. A. Rubel, W. A. Squires \& B. A. Taylor. Irreducibility of certain entire functions with applications to harmonic analysis. Ann. Math. 108 (1978), 553-567. 